\title{
A SIMPLE INTERPOLATION PROCEDURE FOR DIMENSIONAL CROSSOVER PHENOMENA
}

\author{
L. De Cesare* , L. Craco ${ }^{\dagger}$, I. Rabuffo*, D. I. Uzunov ${ }^{\ddagger}$ \\ *Dipartimento di Scienze Fisiche "E. R. Caianiello", Università di Salerno, I-84081 Baronissi, Salerno, Italy, and Istituto \\ Nazionalle per la Fisica della Materia, Unità di Salerno, Italy \\ ${ }^{\dagger}$ Max-Planck-Institut für Physik Komplexer Systeme, Nöthnitzer Strasse 38, D-01187 Drezden, Federal Republic of Germany \\ ${ }^{\ddagger}$ CPCM Laboratory, G. Nadjakov Institute of Solid State Physics, Bulgarian Academy of Sciences, BG-1784 Sofia, Bulgaria \\ (Received April 24, 1998)
}

\begin{abstract}
A method of investigation of dimensional crossover phenomena in the theory of phase transitions is illustrated for the finite-size crossover. The dimensional crossover phenomena in systems with quenched extended impurities and inhomogeneities as well as the classical-to-quantum crossover at extremely low temperatures are also discussed. The fundamental interrelationship between the effective dimensionality and the ratio of the relevant characteristic lengths of the system is established and applied to the description of dimensional crossover phenomena.

Key words: finite size scaling, phase transition, renormalization group, dimensional crossover, quantum critical phenomena.
\end{abstract}

PACS number(s): 05.70.Jk, 64.60.Ak, 64.60.Fr, 68.35.Rh

\section{PURPOSES}

A simple way for the description of the finite-size dimensional crossover (FSC) $[1,2]$ in the theory of critical phenomena [3] of thin films is presented. The method is based on an approximate interpolation of the lattice summation by an integration in spatial dimensionalities less than unity. The powerful tool of investigation of the FSC is the $q$-space renormalization-group (RG) method [3]. Further we discuss mainly the application of the RG to the FSC in slabs (see preceding works $[4,5]$ ). The consideration is based on the notion of the interrelationship between the effective spatial dimensionality $D_{\text {eff }}$ of the critical fluctuations and the ratio $y=\left(L_{0} / \xi\right)$ of the thickness $L_{0}$ of the slab to the correlation length $\xi \sim\left(\left|T-T_{c}\right| / T_{c}\right)^{-1 / 2}$. This notion is familiar, but here for the first time it is considered in an explicit form with the intention to exploit it in the description of the FSC as a smooth transition from a $(D-1)$ - to a $D$-dimensional asymptotic critical behaviour (Sections IV-IX). Moreover, the term "effective spatial dimensionality" in the sense defined below is introduced for the first time in the present report. The fundamental problem for the interrelationship between the effective dimensionality $D_{\text {eff }}$ and the ratio $y$ is examined in Sections V, VI and XI. Applications of the results to other crossover phenomena in quantum systems and systems with quenched impurities are discussed in Section X.

\section{MODEL}

In order to present the idea and the purpose of this note we choose the example of the $\phi^{4}$ - fluctuation Hamiltonian $\left(\mathcal{H}=H / T, k_{B}=1\right)$ of the second order phase transitions,

$$
\mathcal{H}=\frac{1}{2} \int d^{D} x\left\{(\nabla \phi)^{2}+r \phi^{2}+2 u \phi^{4}\right\}
$$

which is often used in the studies of the FSC [4,5]. In Eq. (1), $\phi(\mathbf{x})=\left\{\phi_{1}(\mathbf{x}), \ldots, \phi_{n}(\mathbf{x})\right\}$ is the $n$-component fluctuation field (order parameter), $D$ is the spatial dimensionality, $r \sim\left(T-T_{c}\right)$, and $u$ is a positive parameter for all values of the temperature $T$ near the critical temperature $T_{c},\left(\left|T-T_{c}\right| / T_{c}\right)<1$, where the field model (1) is valid.

\section{GEOMETRY}

For our aims we shall consider a $D$-dimensional FS system having a "slab" geometry, where the thickness $L_{0}$ of the $D$-dimensional hyperslab is the "small" (finite) size and the other $(D-1)$ dimensions $L_{i}, i=1, \ldots, D-1$, are supposed to be "infinite". In fact, the important point is that the ratio $y=\left(L_{0} / \xi\right)$ of the finite size $L_{0}$ and the correlation length $\xi=r^{-1 / 2}$ can take any value from zero to infinity depending on the variations of $L_{0}$ and $T$. In contrast to the "finite" thickness $L_{0}$, the other dimensions $L_{i}$ of the slab are called "infinite" because they can be always considered larger than the correlation length $\xi$, including the case of $\xi \rightarrow \infty$ for $T \rightarrow T_{c}$. For $D=3$ we have a real slab. With respect to the critical properties, the slab behaves as a quasi- $2 D$ film for $y \ll 1$ and exhibits a typical critical behaviour of $3 D$ systems for $y \gg 1$; here " $2 D$ " and " $3 D$ " denote two-dimensional and three-dimensional systems, respectively.

Other FS geometries can be also considered [2], but we shall focus our attention on the FSC in slabs. We shall investigate the $2 D-3 D$ dimensional crossover of the critical behaviour of $3 D$ FS systems and, more generally, the $(D-1)-D$ dimensional crossover in $D$-dimensional systems. The dimensionality $(D-1)$ is assumed larger than the lower critical dimensionality $D_{L}$ [3]. This condition 
is necessary to ensure the second order phase transition and critical phenomena of the usual type in both $D$ and $(D-1)$ spatial dimensionalities.

It is convenient to write the $D$-dimensional wave vector $q$ in the form of $q=\left(k_{0}, \mathbf{k}\right)$, where $k_{0}=\left(2 \pi n_{0} / L_{0}\right)$ with $n_{0}=0, \pm 1, \ldots$, and $k_{i}=2 \pi n_{i} / L_{i}$ with $n_{i}=0, \pm 1, \ldots$ In the case of lattice structures, the wave vector components $q_{\nu}(\nu=0,1, \ldots, D-1)$ are confined in the first Brillouin zone $\left(-\pi / a<q_{\nu}<\pi / a\right)$ but this condition can be safely ignored because the correlation length $\xi$ in our problem is always much larger than the lattice constant $a[6]$. Because of this and having in mind that the model (1) describes only quasimacroscopic phenomena, the upper cutoff $\Lambda$ of all wave vector components $k_{\nu}$ is given by $\Lambda \ll \pi / a$ and all short-range phenomena described by the modes $|q|<\Lambda \ll(\pi / a)$ are neglected [6]. Thus we are left with the free choice to use the natural upper cutoff $\Lambda$ or to ignore it. As we shall apply the Wilson-Fisher variant of the RG, it seems convenient to keep the cutoff of the $(D-1)$ components $k_{i}$ and to neglect it for the component $k_{0}$. The latter choice ensures a simplification in the summation over $k_{0}$ in our calculations. In the remainder of this paper we shall use units of lengths, for which $\Lambda=1$.

\section{PERTURBATION INTEGRALS}

Now let us discuss the self-energy perturbation contributions to the RG equations [3] with the rescaling factor $b>1$ for the $(D-1)$ wave numbers $k_{i}$ and without any rescaling for the wave number $k_{0}$. Such a RG scheme exists and yields excellent results [5]. The one-loop contributions including all Hartree terms for the self-energy function and the interaction constant $u$ are calculated by simple lattice sums of the type

$$
A_{m}(r, b)=\frac{1}{V_{d}} \sum_{\mathbf{k}} B_{m}(k, r),
$$

where

$$
B_{m}(k, r)=\frac{1}{L_{0}} \sum_{\mathbf{k}_{0}} \frac{1}{\left(k_{0}^{2}+k^{2}+r\right)^{m}}
$$

$m=1,2, \ldots, V_{d}=\left(L_{1} \ldots L_{D-1}\right)=V_{D} / L_{0}$, and $k=|\mathbf{k}|$. While the $\mathbf{k}$-summation in (2) can be always replaced by a k-integration because of the "infinite" dimensions $L_{i}$, the summation over $k_{0}$ in (2) cannot be transformed to an integration unless $L_{0} \gg \xi$.

As $A_{m+1}=-\left(\partial A_{m} / \partial r\right)$ for all $m \geq 1$, one needs to know only the sum $B_{1}$. After the direct summation in (3) we obtain the simple transcendental function

$$
B_{1}=\frac{L_{0}}{2 y(k)} \operatorname{cth}\left[\frac{y(k)}{2}\right]
$$

where $y(k)=L_{0}\left(k^{2}+r\right)^{1 / 2}$ and, obviously, $y(0) \equiv y=$
$L_{0} / \xi$ (a Gaussian approximation for $\xi$ ).

It is seen from Eqs. (2)-(4) that the quantities $A_{m}$ will exhibit power law dependences on $y$ only in the limiting (asymptotic) cases $y \rightarrow 0$ (quasi-2D slabs) and $y \rightarrow \infty$ (3D systems). In the intermediate cases $(0<y<\infty)$, the functions $A_{m}(y)$ are given by infinite series in the powers of $y$. The intermediate cases can be treated by replacing the $k_{0}$-summation by an integration with the help of the Euler-Maclaurin summation formula [7], but in this approach one again obtains series in the inverse powers of $y$ as corrections to the corresponding integrals. The corrections can be neglected only for $y \gg 1$, i. e. when the direct replacement of the summation with an integration is possible. Therefore, one can easily use the power law behaviour of the quantities $A_{m}$ in the limiting cases in order to obtain scaling invariant RG equations for quasi$2 D$ and $3 D$ systems but it seems difficult to solve the same problem for the intermediate cases $(0<y<\infty)$. The reason is that the $R G$ equations contain transcendental functions like the function (4) and its derivatives. The direct way to solve the problem for the description of the dimensional crossover for all the values of the ratio $y$ is to use a numerical analysis of the corresponding transcendental RG equations [5]. Here we shall consider an alternative approach which leads to an approximate solution of the problem.

\section{INTEGRATION IN DIMENSIONALITIES LESS THAN UNITY}

Consider the following $\delta(\leq 1)$-dimensional integration as a substitution of the usual $k_{0}$-summation:

$$
\frac{1}{L_{0}^{\delta}} \sum_{k_{0}} \rightarrow \int \frac{d^{\delta} x}{(2 \pi)^{\delta}} \equiv \frac{S_{\delta}}{(2 \pi)^{\delta}} \int_{0}^{\infty} d x \cdot x^{\delta-1}
$$

where $S_{\delta}=2 \pi^{\delta / 2} / \Gamma(\delta / 2)$ is the area of the unit $\delta$ dimensional sphere $(0<\delta<1)$. For $\delta=1$, correspondence $(5)$ yields the usual rule of the transition from a summation to an integration.

The correspondence (5) seems to be a generalization of the usual rule. However, this correspondence is not exact and, therefore, it should be considered with some caution. The limit $\delta \rightarrow 0$ in the last integral in (5) (in spherical coordinates) should be taken with a special attention because of the divergency of the gamma function $\Gamma(\delta / 2)$.At first one should perform the integration over $x$ of the integrand, say, $x^{+0} f(x) / x$, and then to take the limit $\delta \rightarrow 0$. Usually, the integrands $[\sim f(x)]$ that appear by the perturbation series are such that no divergences arise in the final results.

In order to connect the trick (5) with the physical picture of the FSC, we shall remember that all $\left(k_{0} \neq 0\right)-$ terms in (3) are negligible for $y \ll 1$, and, hence, $B_{1}=1 / L_{0}\left(k^{2}+r\right)$ for $y \rightarrow 0$. The same result for $B_{1}$ follows from Eq. (4) for $y \rightarrow 0$ and from the integration of $f(x)=L_{0}^{\delta-1} /\left(x^{2}+k^{2}+r\right)$ in integral (5) in the limit $\delta \rightarrow 0$. Therefore, there is a relationship $\delta(y)$ between 
the partial dimensionality $\delta$ and the ratio $y$ such that the function $\delta(y) \rightarrow 0$ for $y \rightarrow 0$. Taking the limit $y \rightarrow \infty$ in (4) and the limit $\delta \rightarrow 1$ in (5) one obtains again the same result for $B_{1}$, namely, $B_{1}=1 / 2\left(k^{2}+r\right)^{1 / 2}$. Thus the function $\delta(y)$ tends to unity for $y \rightarrow \infty$.

\section{EFFECTIVE DIMENSIONALITY}

The exact correspondence of the results for $B_{m}$ from (4) and (5) in the two asymptotic cases $(y=0, \delta=0)$ and $(y=\infty, \delta=1)$ brings about the supposition that the function $\delta(y)$ with the mentioned asymptotic values exists and is related to the effective spatial dimensionality

$$
D_{\mathrm{eff}}(y)=d+\delta(y)
$$

where $d=D-1$. This conclusion is completely consistent with the notion that the asymptotic critical behaviour, exhibited by the system, will smoothly change from a $(D-1)$ - to $D$-dimensional critical behaviour when the ratio $y$ varies from zero to infinity. This means that the spatial dimensionality effectively changes by increasing its values smoothly from $(D-1)$ to $D$ when $y$ varies from zero to infinity and this is expressed by Eq. (6). Another problem is the derivation of a reliable formula for the dependence $D_{\text {eff }}(y)$. This fundamental task is briefly discussed in Section XI.

\section{APPROXIMATE INTERPOLATION}

The ideas mentioned above can be used for another task of practical interest. One may try to approximate sum (3) for $B_{1}$ with the integral

$$
B_{m} \rightarrow B_{m}^{\prime}=L_{0}^{\delta-1} \int \frac{d^{\delta} x}{(2 \pi)^{\delta}} \frac{1}{\left(x^{2}+k^{2}+r\right)^{m}}
$$

for all the values of $y \in(0, \infty)$ and, hence, for $\delta \in(0,1)$. It must be emphasized that substitution (7) is exact only for the limiting cases $y=0$ and $y=\infty$. This means that one may consider the correspondence between the sum (4) and integral (7) only as an approximation to the description of the intermediate cases $0<\delta<1$. For $\delta \sim 0$ and $\delta \sim 1$ this approximation leads to negligible errors in the calculation of the sum (4) with the help of $(7)$. The substitution $B_{m} \rightarrow B_{m}^{\prime}$ for the intermediate values of $\delta$ and $y$ leads to results that need substantial corrections. The latter have not been investigated at all and the problem requires a special attention. Despite the obvious approximation introduced by the substitution of $B_{m}$ with $B_{m}^{\prime}$ one may try to use the integral (7) in order to obtain an approximate interpolation between the $2 D$ and $3 D$ asymptotic critical regimes.

\section{RENORMALIZATION GROUP}

The conventional RG treatment of the FSC has been presented in details in the preceding works (see, e.g., [5]). The perturbation integrals like $A_{m}$ have been calculated by a direct summations over $k_{0}$; see, e.g., Eq. (4). Using the alternative given by (5) and (7) we may choose two almost equivalent variants, i. e.

(i) to consider $A_{m}$ as $(d+\delta)$-dimensional integrals

$$
A_{m}(r, b)=L_{0}^{\delta-1} \int \frac{d^{d+\delta} q}{(2 \pi)^{d+\delta}} \frac{1}{\left(q^{2}+r\right)^{m}}
$$

with $d=D-1, q=(x, \mathbf{k})$, which makes the mathematical problem equivalent to that for extended random impurities [8], or

(ii) to consider $A_{m}$ as $(d, \delta)$-integrals:

$$
A_{m}(r, b)=L_{0}^{\delta-1} \int \frac{d^{d} k}{(2 \pi)^{d}} \int \frac{d^{\delta} x}{(2 \pi)^{\delta}} \frac{1}{\left(x^{2}+k^{2}+r\right)^{m}}
$$

The variant (ii) directly follows from the original scheme of treatment [5], where one must perform first the $k_{0}-$ summation and then to accomplish the $\mathbf{k}$-integration. We shall demonstrate that the variants (i) and (ii) lead to the same results for the FSC.

Using standard calculations [3,5], and the notations $J_{\delta}(r)=A_{1}(r, b) L_{0}^{1-\delta}, v=u L_{0}^{\delta-1}$, the $\mathrm{RG}$ relations for the variant (i) can be derived and written in the form:

$$
r^{\prime}=b^{2}\left[r+4(n+2) v J_{\delta}(r)\right]
$$

$$
v^{\prime}=b^{4-(\delta+d)}\left\{v-4(n+8) v^{2}\left[-\partial J_{\delta}(r) / \partial r\right]\right\},
$$

where the upper critical dimensionality is obviously $D_{\mathrm{eff}}^{(U)}=(d+\delta)=4$. This result for the upper effective dimensionality $D_{\text {eff }}^{(U)}$ follows from the scaling factor in Eq. (11) and from the value of the integral $\left[-\partial J_{\delta}(r) / \partial r\right]$ for $r=0$. The latter integral is equal to $K_{(d+\delta)} \ln b$, where $K_{(d+\delta)}=S_{(d+\delta)} /(2 \pi)^{d+\delta}$.

The above results demonstrate a straightforward $d \rightarrow$ $D_{\text {eff }}=(d+\delta)$ dimensional crossover described by the $\epsilon$-expansion, where $\epsilon=4-(d+\delta)=4-D_{\text {eff }}$. Here $\delta$ is not an expansion parameter like $\epsilon$ but takes its values in the whole interval $[0,1]$. All results for the fixed point (FP) coordinates and the critical exponents correspond to the universality class $\left(n, D_{\text {eff }}\right)$. These quantities are expressed by the expansion parameter $\epsilon=4-D_{\text {eff }}$ rather than by the usual ones: $(\epsilon=4-d$, or $\epsilon=4-D)$; see Ref. [5]. The latter result is a direct consequence of the supposition that the exact Eqs. (2)-(4) can be substituted by Eq. (9). The difference between the standard $[4,5]$ and the present studies of the FSC is that the present $\epsilon$-expansion is defined with the help of the 
effective dimensionality $D_{\text {eff }}$ instead of the geometrical dimensionalities $(D$ or $d$ ).

The investigation by the scheme (i) can be continued by using $\delta$ as a small parameter: $\exp (-\delta) \approx 1-\delta$. Within this scheme we have performed a double $(\epsilon=4-d, \delta)-$ expansion. This treatment of Eqs. (10) and (11) yields again that the FPs and the critical exponents are exactly those for the standard universality class $(n, D)$ with the only difference that $D$ should be changed with $D_{\text {eff }}=d+\delta$. All FPs, stability and critical exponents are represented by $(\epsilon-\delta)=4-D_{\text {eff }}$. A special feature of this expansion is that the results are valid for $\epsilon>\delta$. The latter condition ensures the stability of the nontrivial FP for $(\epsilon-\delta)>0$, i.e. for $D_{\text {eff }}<4$. This property of the $(\epsilon, \delta)$-expansion within the framework of the variant (i) is a disadvantage with respect to the direct $\epsilon=\left(4-D_{\text {eff }}\right)$-expansion discussed above.

The established restriction $\epsilon>\delta$ should be taken into account when performing the double $(\epsilon, \delta)$-expansions in treating various problems. A special attention should be paid to extrapolations and interpretations of the results from such expansions for real dimensionalities of thick films $(\delta \sim 1)$.

The RG within the framework of the variant (ii) can be performed both with or without a rescaling of the variable $x$, i. e. $k_{0}[5]$. The rescaling requires a finite cutoff $\Lambda=1$ of the wave number $k_{0}$, too, but the calculation of the perturbation integrals is quite hard and can be analytically performed for $\epsilon=\delta=0$. In this case the difficulty lies in the divergence of the factor $K_{\delta} \sim \Gamma^{-1}(\delta / 2)$ for $\delta \sim 0$. This can be avoided by introducing an expansion around the value $\delta=1$ ( $3 D$ system) with the expansion parameter $\delta^{\prime}=1-\delta \ll 1$.

In order to keep our consideration far from such difficulties we shall use a more rational scheme of RG, in which the initial rescaling of the wave component $k_{0}$ is avoided and the corresponding integration over $x$ has not an upper cutoff [5]. This approach is performed again by the $\epsilon=4-(d+\delta)$ - expansion. The RG transformation in the one-loop approximation yields a simple RG relation for $k_{0}$ (or $L_{0}$ ), namely, $k_{0}^{\prime}=b k_{0}$ or, equivalently, $L_{0}^{\prime}=b^{-1} L_{0}$. The RG recursion relations for $r$ and $u$ will be:

$$
\begin{gathered}
r^{\prime}=b^{2}\left[r+4(n+2) u A_{1}(r, b)\right], \\
u^{\prime}=b^{3-d}\left[u-4(n+8) u^{2} A_{2}(0, b)\right],
\end{gathered}
$$

where $A_{m}(r, b)$ are given by Eq. (9). Performing the integration in $A_{m}(r, b)$ within the variant (ii) to the required accuracy $[3,5]$, we have

$$
A_{1}(r, b) \approx A_{1}(0, b)-r A_{2}(0, b),
$$

$$
\begin{gathered}
A_{1}(0, b)=\frac{\tau(\delta)}{(2-\delta)} K_{4-\delta} L_{0}^{\delta-1}\left(1-b^{-2}\right) \\
A_{2}(0, b)=\tau(\delta) K_{4-\delta} L_{0}^{\delta-1} \ln b
\end{gathered}
$$

where $\tau(\delta)=\Gamma(1-\delta / 2)(1-\delta / 2)(4 \pi)^{\delta / 2}$. The $L_{0^{-}}$ dependence in the r.h.s. of these RG relations implies a change of the interaction parameter from $u$ to $v=u L_{0}^{\delta-1}$.

The standard RG analysis of Eqs. (12) and (13) reveals the usual $(n, d+\delta)$ universality class [3] described by the Heisenberg FP for all $\delta \in[0,1]$. In comparison with the usual case $(\delta=1)$, the coordinates $r_{H}$ and $v_{H}$ of the Heisenberg FP have an extra $\delta$-dependence given by the factors $2 /(2-\delta)$ and $\left[\tau(\delta) K_{4} / K_{(4-\delta)}\right]$, respectively. Therefore, the FP coordinates in the variant (ii) depend on $\delta$. This fact has no consequences for the critical behaviour. The values of the stability and critical exponents are given by the usual formulae of the $\epsilon$-expansion [3], where $\epsilon=4-D_{\text {eff }}$.

The above results within the variant (ii) prove the FSC for all the values of $\delta$ (and $y$ ). Of course, the proof is given within the one-loop approximation, as it has been done in the variant (i).

\section{DISCUSSION OF RESULTS}

It has been shown that variants (i) and (ii) lead to the same results. Using two variants of calculation, (i) and (ii), it has been proven to one-loop order, that the FSC occurs for any $\delta$. The proof of this basic result could be done up to the two-loop approximation, too. In particular, this can be more easily accomplished within the variant (i) of calculation. Note, that the Wilson-Fisher recursion relations cannot be derived in higher than the twoloop approximation, and the results presented here cannot be extended beyond this limitation of the method.

The next orders in the loop expansion can be investigated with other variants [9] of the $\mathrm{RG}$. The $\beta$-function for the $\epsilon=\left(4-D_{\text {eff }}\right)$ - expansion can be calculated in the same way as it is known for the usual case of $\epsilon=(4-d)-$ expansions within the $\phi^{4}$-theory of infinite systems [9]. This is the case for the variant (i). It seems that one does not need to prove the last statement order by order in the loop expansion.

The above RG results for the FSC are valid for $(d+\delta)>2=D_{L}-$ the lower critical dimensionality. Therefore, the results cannot be extrapolated for $2 D$ dimensional films (monoatomic layer; $d=2, \delta=0$ ). This is the general disadvantage of the field-theoretical RG methods. The RG results presented here are, however, reliable in predictions for the real case described by $d=(D-1)=2$ and $\delta>0$ (quasi-2 $D$ films). This is the case of thin or ultrathin films where $0<\delta \ll 1$. Certainly, the present consideration covers a wide class of film geometries: from ultrathin films $(\delta \ll 1)$ to thick films (slabs), where $\delta \sim 1$. 


\section{CLASSICAL-TO-QUANTUM CROSSOVER AND IMPURITY EFFECTS}

The above consideration can be applied to other dimensional crossover phenomena. We shall consider briefly the dimensional classical-to-quantum crossover (CQC) $[3,10]$. In this case, the $d$-dimensional quantum system has a standard classical asymptotic critical behaviour at all finite critical temperatures $T_{c} \neq 0$ but this behaviour changes in the quantum limit $\left(T_{c} \rightarrow 0\right)$ and, in certain cases, it becomes equivalent to the $(d+z)-$ dimensional classical $\left(T_{c} \neq 0\right)$ critical behaviour of the same system; $z$ is the dynamical critical exponent. This CQC is given by $d \rightarrow(d+z)$. The responsible effects are the quantum correlations which become relevant for low temperatures. In order to treat the $\mathrm{CQC}$ within the above scheme we must change the variable $L_{0}$ with the thermal wave length $\lambda(T) \sim(1 / T)^{1 / 2}$; for certain quantum statistical models, $\lambda \sim 1 / T^{\sigma}$ with a positive $\sigma \neq(1 / 2)[3,10]$.

In order to give an example, let us consider a wide class of quantum statistical models of Bose fields $\phi\left(\omega_{l}, \mathbf{k}\right)$ described by the (bare) correlation function $G=\left\langle\phi^{*}\left(\omega_{l}, \mathbf{k}\right) \phi\left(\omega_{l}, \mathbf{k}\right)\right\rangle$ of the form

$$
G=\frac{1}{\omega_{l}^{2}+k^{2}+r},
$$

where $\omega_{l}=2 \pi l T$ with $l=0, \pm 1, \ldots$ and $\hbar=1$ is the (Bose) Matsubara frequency. In such quantum field models, the thermal wave length $\lambda(T)$ is proportional to $1 / T$ [3]. The formal change of $T$ in the $\omega_{l}^{2}$-term in (17) with $1 / L_{0}$ yields the correlation function for our previous problem. Thus the formal correspondence $L_{0} \leftrightarrow 1 / T$ makes possible to describe the several most important features of the FSC and the CQC by using almost the same ways of treatment.

The generalization of our discussion of the FSC to the CQC in other quantum models can be also made, but owing to specific features the analogy between the $\mathrm{CQC}$ in certain quantum models and the FSC in slabs is not straightforward. Let us clarify this point. The quantum $\phi^{4}$-models with the correlation function (17) have a dynamical critical exponent $z$ equal to unity (in Gaussian approximation) $[3,10]$. This is the reason for the direct correspondence between the $\mathrm{CQC}$ in these systems and the FSC in slabs. For certain quantum models [3,10] the dynamical critical exponent $z$ in the Gaussian approximation is different from unity and then another correspondence between the partial dimensionality $\delta$ in the FS problem and the corresponding quantity $\delta_{q}$ in the quantum problem will be valid, namely, $\delta_{q}=z \delta$. Moreover, the description of the CQC has additional specific problems because of the fact, that the basic quantum models like certain Bose systems at $T=0$ exhibit a special Gaussian-like critical behaviour rather than satisfy the CQC; see G. Busiello and L. De Cesare (1980), D. I. Uzunov (1981), and M. J. Schakel (1995) in Ref. [10].

The method considered in this paper has been applied [11] to thin impure films with quenched disorder.
The quantum models with quenched disorder has been considered by G. Busiello, L. De Cesare and I. Rabuffo as well as by E. R. Korutcheva and D. I. Uzunov [12] including the effect of the CQC in the quantum limit $T_{c} \rightarrow 0$. It is shown by these authors that the quantum critical behaviour is unstable towards the effect of quenched disorder and this problem has been further studied by A. Schakel [13] for Bose systems and by T. R. Kirkpatrick and D. Belitz [14] for impure quantum antiferromagnets. The result of the last two studies is that the stability of the quantum impure behaviour can be restored by double $(\epsilon, \delta)$-expansions of the type considered in the present paper. In spatial dimensionalities above the upper quantum critical dimensionality $d_{q}^{(U)}=(4-z)$ the thermal fluctuation interactions are weak in comparison with the effects of the quenched disorder and the latter acts like a disorder in systems of free (noninteracting) fluctuations (Gaussian model). The introduction of small $\delta \sim z$ makes possible a consideration, in which the difference between the classical and the quantum upper critical dimensionalities given by the dynamical exponent $z$, becomes one of the expansion parameters. Then one may consider the interplay between the disorder effects and the thermal fluctuation interactions. This approach yields a stable FP of the RG equations and a new quantum critical behaviour $[13,14]$ but the question is whether this can be physically justified in the quantum limit $T \rightarrow 0$ where the thermal fluctuations are absent. Moreover, as it has been established in this paper, the extensions of the small parameter $\delta_{q} \sim z$ to values of the order of unity should be made only under the condition $\epsilon>\delta_{q}$.

\section{THE RELATION $D_{\text {eff }}(y)$}

Our investigation is based on the physical notion that there is a fundamental interrelationship between the ratio $y$ of relevant lengths and the effective spatial dimensionality $D_{\mathrm{eff}}=(d+\delta) \neq D$ of the asymptotic critical behaviour. We have introduced the term effective dimensionality to describe the (effective) dimensionality of the strongly interacting thermal (classical) fluctuations rather than to attach such a term to the system itself. This new term has been used in our practical calculations and in the interpretation of the results. However, we have not succeeded in obtaining the dependence $D_{\text {eff }}(y)$ from our construction of the integration in partial dimensionalities $\delta$. The reason is that (3) and (7) do not give the same value for the quantity $B_{m}$ for all the cases where $0<\delta<1$ and, hence, the exact Eq. (3) and the approximate correspondence $(7)$ cannot be equated in order to find a functional equation for $\delta(y)$.

However, the interrelation of interest can be evaluated approximately. Suppose, that for any given value of $y$, i.e. for any $L_{0}$ and $\xi$, there is a value of $\delta \in[0,1]$, for which the value of $B_{1}$ from (3) is extremely close to the value of $B_{1}^{\prime}$ from (7). The values $B_{m}$ and $B_{m}^{\prime}$ will coincide only for the trivial cases of $(\delta=1, y=\infty)$ and $(\delta=0, y=0)$, but their nonzero difference in the intermediate cases could be minimal for some function $\delta(y)$. Following this idea, 
one may find an approximate relationship between $\delta$ and $y$. It may be expected, that $\delta(y) \approx 1$ for $y>1$, and $\delta(y) \approx 0$ for $y<1$ as well as that the values of $\delta(y)$ will be substantially different from unity or zero only in a close vicinity of the value $x=1$.
[1] M. E. Fisher, in Critical Phenomena, edited by M. S. Green [Proceedings of the 5th Enrico Fermi Summer School, Varenna, Italy] (Academic Press, London, 1971), p. 1; M. E. Fisher and M. N. Barber, Phys. Rev. Lett. 28, 1512 (1972).

[2] For a review, see, e.g., M. N. Barber, in Phase Transitions and Critical Phenomena, edited by C. Domb and J. L. Lebowitz (Academic Press, London, 1983).

[3] See, e.g., D. I. Uzunov, Introduction to the Theory of Critical Phenomena (World Scientific, Singapore, 1993).

[4] J. A. Bray and M. A. Moore, J. Phys. A 11, 715 (1978); E. Brezin, J. Phys. (Paris) 43, 15 (1982); E. Brezin and J. Zinn-Justin, Nucl. Phys. B 257, 867 (1985); D. O'Conner and C. R. Stephen, Nucl. Phys. B 360, 397 (1985).

[5] D. I. Uzunov and M. Suzuki, Physica A 216, 489 (1995).

[6] D. I. Uzunov, in Lectures on Cooperative Phenomena in Condensed Matter, edited by D. I. Uzunov (Heron Press, Sofia, 1996), p. 46.

[7] See, e.g., G. A. Korn and T. M. Korn, Mathematical Handbook for Scientists and Engineers (McGraw-Hill, New York, 1968).

[8] S. N. Dorogovtsev, Phys. Lett. A 76, 169 (1980); D. Boyanovsky and J. L. Cardy, Phys. Rev. B 26, 154 (1982);
I. D. Lawrie and V. V. Prudnikov, J. Phys. C 17, 1655 (1983); A. Weinrib and B. I. Halperin, Phys. Rev. B 27, 413 (1983).

[9] J. Zinn-Justin, Euclidian Field Theory and Critical Phenomena (Oxford University Press, Oxford, 1989); Second and Third Editions: 1993, 1996.

[10] J. A. Hertz, Phys. Rev. B 14, 1165 (1976); G. Busiello and L. De Cesare, Phys. Lett. A 77, 177 (1980); D. I. Uzunov, Phys. Lett. A 87, 11 (1981); A. M. J. Schakel, Nucl. Phys. B 453, 705 (1995); A. Caramico D'Auria, L. De Cesare and I. Rabuffo, Physica A 225, 363 (1996); A. Caramico D'Auria, L. De Cesare, U. Esposito, and I. Rabuffo, Physica A 243, 152 (1997); A. M. J. Schakel, Boulevard of Broken Symmetries, a preprint of Freie University of Berlin (1997).

[11] L. De Cesare, L. Craco, I. Rabuffo, I. P. Takov, and D. I. Uzunov, 1998 (unpublished).

[12] E. R. Korutcheva and D. I. Uzunov, Phys. Lett. A 106, 175 (1984) [Preprint, ISSP-BAS, 1983]; G. Busiello, L. De Cesare and I. Rabuffo, Phys. Lett. A 102, 41 (1984).

[13] A. M. J. Schakel, Phys. Lett. A 224, 287 (1997).

[14] T. R. Kirkpatrick and D. Belitz, Phys. Rev. Lett. 76, 2571 (1996). 